\title{
Quantifying Between-Cohort and Between-Sex Genetic Heterogeneity in Major Depressive Disorder
}

Maciej Trza skowski1, Divya Mehta 1,2, Wouter Peyrot³, Da vid Hawkes4, Da niel Davies5, David

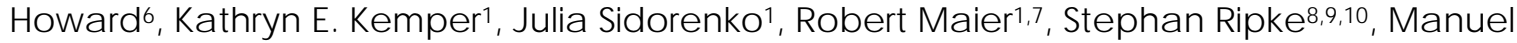
Mattheisen 11,12, Major Depressive Disorder Working Group of the Psychiatric Genomics Consortium, Bemhard TBaune ${ }^{13}$, Hans J. Grabe ${ }^{14}$, Andrew C Heath ${ }^{15}$, Lisa J ones ${ }^{16}$, lan J ones ${ }^{17}$, Pamela AF Madden ${ }^{15}$, Andrew M. Mc Intosh ${ }^{18,19}$, Gerome Breen ${ }^{20,21}$, Cathryn M. Lewis20,21, Anders D. Børg lum 12,23, Patrick F. Sullivan 24,25,26, Nic holas G. Martin27, Kenneth S. Kendler28, Douglas F. Levinson ${ }^{29}$, Na omi R. Wra y1,30†

IInstitute for Molec ular Bioscience, The University of Queensland, Brisbane, Queensland, Australia.

2School of Psychology and Counselling, Queensland University of Tec hnology, Brisba ne, Australia.

3Department of Psychiatry, Vrije Universiteit Medic al Center and G G Zin Geest, Amsterdam, The Netherlands.

${ }^{4} \mathrm{AGRF}$, The University of Queensland, Brisbane, Queensland, Austra lia.

${ }^{5}$ Department of Psychiatry, Behavioural and Clinic al Neuroscience Institute and

Developmental Psychia try, Cambridge University, UK.

6Division of Psychia try, University of Ed inburgh, Ed inburgh, EH10 5HF, UK.

${ }^{7}$ Ana lytic and Transla tional Genetic s Unit, Massac husetts General Hospital and Ha rva rd

Medical School, Boston, Massa chusetts, USA.

8Medic al and Population Genetics, Broad Institute, Cambridge, MA, USA.

${ }^{9}$ Ana lytic and Translational Genetic s Unit, Massa c husetts G eneral Hospital, Boston, MA, USA. 10Department of Psychiatry and Psychothera py, Universitätsmedizin Berlin Campus Cha rité Mitte, Berlin, Germany.

${ }^{11}$ Department of Biomedic ine, Aa rhus University, Aa rhus, Denmark.

12iPSYCH, The Lundbeck Foundation Initiative for Integrative Psychiatric Research, Denmark. 13Department of Psyc hiatry, The University of Melboume.

${ }^{14}$ Department of Psychiatry and Psychotherapy, University Medic ine Greifswa ld, Germany. ${ }^{15}$ Department of Psyc hiatry, Wa shington University in Sa int Louis Sc hool of Medic ine, Sa int Louis, MO, US.

${ }^{16 I n s t i t u t e ~ o f ~ H e a l t h ~ \& ~ S o c ~ i e t y, ~ U n i v e r s i t y ~ o f ~ W o r c ~ e s t e r, ~ W o r c ~ e s t e r, ~ U K ~}$

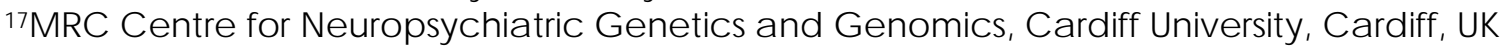

${ }^{18}$ Division of Psyc hiatry, University of Ed inburgh, Ed inburgh, UK.

${ }^{19}$ Centre for Cognitive Ageing and Cognitive Epidemiology, University of Ed inburgh,

Edinburgh, UK.

20MRC Social Genetic and Developmental Psychiatry Centre, King's College London, London, UK.

${ }^{21} \mathrm{NIHR}$ BRC for Mental Health, King's College London, London, UK.

22Department of Medical and MolecularGenetics, King's College Lond on, London, UK.

${ }^{23}$ Department of Biomedic ine and iSEQ-Centre for Integrative Sequenc ing, Aarhus University, Denmark.

${ }^{24}$ Department of Medical Epidemiology and Biostatistic s, Ka rolinska Institutet, Stockholm, Sweden.

${ }^{25}$ Department of G enetics, University of North Ca rolina at Chapel Hill, Chapel Hill, NC, USA.

26Department of Psyc hiatry, University of North Ca rolina at Chapel Hill, Cha pel Hill, NC, USA.

This is the author manuscript accepted for publication and has undergone full peer review but has not been through the copyediting, typesetting, pagination and proofreading process, which may lead to differences between this version and the Version of Record. Please cite this article as doi: 10.1002/ajmg.b.32713 
${ }^{27}$ Genetic s and Computational Biology, Q IMR Berghofer Medic al Research Institute, Brisba ne, Queensland, Australia.

28Department of Psyc hiatry, Virginia Commonwealth University, Richmond, VA, USA.

${ }^{29}$ Psychiatry and Behavioral Sciences, Stanford University, Stanford, CA, USA.

${ }^{30}$ Q ueensland Bra in Institute, The University of Queensland, Brisbane, Queensland, Australia.

t Comesponding author: naomi.wray@uq.edu.au

This article is protected by copyright. All rights reserved. 
ABSTRACT

Major depressive disorder (MDD) is clinically heterogeneous with prevalence rates twice as high in women as in men. There are many possible sources of heterogeneity in MDD most of which are not measured in a sufficiently comparable way across study samples. Here, we assess genetic heterogeneity based on two fundamental measures, between-cohort and between-sex heterogeneity. First, we used genome-wide association study (GWAS) summary statistics to investigate between-cohort genetic heterogeneity using the 29 research cohorts of the Psychiatric Genomics Consortium (PGC; N cases $=16,823$, $N$ controls $=25,632$ ) and found that some of the cohort heterogeneity can be attributed to ascertainment differences (such as rec nuitment of cases from hospital vs community sources). Second, we evaluated between-sex genetic heterogeneity using GWAS summary statistics from the PGC, Kaiser Permanente GERA, UK Biobank and the Danish iPSYCH studies but did not find convincing evidence forgenetic differences between the sexes as the genetic comelation between the sexes was not signific antly different from one ( 0.90 s.e. 0.06 ; $\left.p_{H 0: r g=1}=0.096\right)$. We conc lude that there is no evidence that the heterogeneity between MDD data sets and between sexes reflects genetic heterogeneity. Larger sample sizes with detailed phenotypic records and genomic data remain the key to overcome heterogeneity inherent in assessment of MDD.

KFYWORDS: MDD, depression, genetic heterogeneity, sex differences, LD score regression.

\section{INTRODUCTION}

Major Depressive Disorder (MDD) is a common debilita ting disorder with lifetime risk of $\sim 15 \%$ (R. C. Kessler \& Bromet, 2013; Lohoff, 2010). Genetic factors contribute to etiology of MDD with herita bility estimated to be $\sim 37 \%$ (Kendler, Gatz, Gardner, \& Pedersen, 2006; Sullivan, Neale, \& Kendler, 2000) of which about one-third is tracked by common-genetic variants 
(Cross-Disorder Group of the Psychiatric Genomic set al., 2013; Wray et al., 2018). Nongenetic factors also contribute and environmental risk factors include childhood psychological trauma (Chapman et al., 2004; Heim, Newport, Mletzko, Miller, \& Nemeroff, 2008; Vythilingam et al., 2002), social isolation (Bruce \& Hoff, 1994), a nd medic al conditions, such as cardiovasc ular disea se (Fiedorowicz, 2014; Fraguas) ret al., 2007; Huffman, Cela no, Beach, Motiwala, \& J a nuzzi, 2013). Most complex disorders are considered to be heterogeneous at clinical presentation. For MDD, heterogeneity is inherent in the diagnostic framework since diagnosis is a chieved through different combinations of endorsements of at least five out of nine criteria in the context of depressed mood for most of the day every day for two weeks (Dia gnostic and Statistic al Manual of Mental Disorders (DSM) criteria). Heterogeneity in symptom profiles between individuals reflects not only the symptoms endorsed, but for some criteria (those assessing sleep, weight/appetite and psychomotor function) the endorsement can reflect either increase or decrease (or both). It is pla usible that these clinical differences reflect different biologic al pathways. The lack of a biological "gold standard" definition in psychiatric illness is well recognised (Kapur, Phillips, \& Insel, 2012), and a key question for the field is whether genetic heterogeneity underpins phenotypic heterogeneity (Fanous \& Kendler, 2005), and if genome-wide genetic data can support analyses that demonstrate genetic heterogeneity (Han et al., 2016). Here, we assess genetic heterogeneity based on two fundamental measures available to us, between-cohort and between-sex heterogeneity. While non-biologic al factors (such as ascerta inment strategy) could contribute to both between-cohort and between-sex heterogeneity, evidence for between-sex heterogeneity may reflect, at least in part, biologic al differences.

Prevalence rates of MDD in women that are double those of men are consistently reported in epidemiological studies, with lifetime risk approximately 0.2 for females and 0.1 for males

This article is protected by copyright. All rights reserved. 
(Ronald C. Kessler, 2003) Women tend to have younger age of onset, greater comorbidity with panic and other anxiety disorders, whereas men exhib it stronger comorbid ity with alc ohol dependence or abuse (Schuch, Roest, Nolen, Penninx, \& de J onge, 2014). Attempts to link the epidemiological differences to biologic al differences have been less consistent. Some twin studies reported signific a ntly higher herita bility in females $(0.42,95 \% \mathrm{Cl}=0.36-0.47)$ than males $(0.29,95 \% \mathrm{Cl}=0.19-0.38)$, and with genetic correlation signific antly different from 1 $(\mathrm{rg} \sim 0.60,95 \% \mathrm{Cl}=0.31-0.99)$ (Kendler et al., 2006). Other studies failed to find differences between sexes (Femandez-Puja ls et al., 2015). Drawing strong conclusions may be confounded by reporting biases as males a re more likely to under-report their symptoms when compared to females (Hunt, Auriemma, \& Cashaw, 2003; Thomic roft et al., 2017).

We use genome-wide association study (G WAS) summary statistics da ta to investigate genetic heterogeneity of MDD. We study between-cohort genetic heterogeneity using data from the 29 independent studies that comprise the wave 2 PGC-MDD study (PG C 29 (Wray et al., 2018)). We a lso investigate genetic heterogeneity by sex using GWAS summa ry statistic S from PG C 29 and three other large data sets. We evaluate between-cohort and betweensex genetic heterogeneity estima tes of SNP-herita bilities and genetic correlations. These estimates of genetic parameters, calculated from genome-wide data, provide single statistic summaries of the data. Spec ific ally, differences in SNP-herita bility estima tes between sa mples could imply real differences in the relative magnitude of genetic risk effect sizes between samples or could reflect biases due to ascerta inment cha racteristic s of the sa mple. In contrast, an estimate of a genetic correlation less than one may reflect differences in the rela tive ordening of genetic risk effects between samples. It is possible for SNP-herita bilities to differ between samples but the genetic correlations to be one.

This article is protected by copyright. All rights reserved. 


\section{MATERIALS \& METHODS}

\section{Between-cohort heterogeneity}

We investigate heterogeneity between cohorts from the PGC Working G roup for MDD (PGCMDD) (Major Depressive Disorder Working Group of the Psychiatric et al., 2013), which comprises 29 cohorts (PGC29, 10 from wave 1 (Major Depressive Disorder Working Group of the Psychia tric et al., 2013) and 19 from wave 2 (Wray et al., 2018)), totalling 16,815 cases (68\% female) and 25,485 controls (51\% female) (Table 1, Supplementary Table 1 ). Cohorts represent individual studies in which cases and controls were imputed together to the 1000 Genomes reference panel (Genomes Project et al., 2010) from a common set of SNPs that had been processed through a common quality control (QC) pipeline (Wray et al., 2018). For the majority of cohorts (but not all), cases and controls were collected by the same research group and were genotyped together on the same genotyping a rray. All 29 case cohorts passed a structured methodological review by MDD assessment experts (DF Levinson and KS Kendler). Cases were required to meet intemational consensus criteria (DSM-IV, Intemational Statistic a I Classific ation of Disea ses (ICD)-9, or ICD-10) (Americ an Psychia tric Association, 1994; World Health Orga nization, 1978, 1992) for a lifetime diagnosis of MDD esta blished using struc tured diagnostic instruments from assessments by tra ined interviewers, clinic ianadministered chec klists, or medic al record review. Nonetheless, there were differences in ascerta inment ac ross cohorts (Supplementary Table 1). For exa mple the RADIANT cohort (rad3) (C. M. Lewis et al., 2010) recruited cases of clinic a lly assessed recurrent MDD, which being more severe have lower lifetime risk $\sim 5 \%$ (McGuffin, Katz, Watkins, \& Rutherford, 1996), compared to community sa mples such as the QIMR cohorts (qi3c, qi6c, qio2) assessed by self-report interview and with lifetime risk $\sim 24 \%$ (Mosing et al., 2009). To capture heterogeneity due to ascerta inment, we coded the 29 cohorts as identified in community, psychiatric outpatient, psychiatric inpatients, or mixed in-/out-patient settings (Supplementary Table 1).

This article is protected by copyright. All rights reserved. 


\section{Between-sex heterogeneity}

We investigate between sex heterogeneity using four large MDD data sets (Table 1). In addition to PGC29, we used the Genetic Epidemiology Research on Adult Health and Aging (GERA) Cohort (Banda et al., 2015) (where electronic medic al rec ords from the Ka iser Permanente healthcare system were used to identify cases as individuals being treated clinic ally for MDD, and controls had no recorded treatment for any psychiatric disorder), the Danish iPSYCH cohort (where national hospital records id entified cases as those ever treated clinic ally for MDD and controls as those who have not), and the volunteer UK Biobank (Bycroft et al., 2018; La ne et al., 2016) (UKB) study. UKB cases were those with either recorded ICD10 codes for MDD (F32, F33) or self-report for seeking treatment for nerves, a nxiety or depression; for detailed description of the "broad depression" definition see reference (Howard et al., 2018)). Exclusions for both cases and controls were those with recorded schizo phrenia, bipolar or mental retardation diagnoses or presc riptions associated with these disorders. Additional exc lusions for controls included those with recorded anxiety, phobic or a utistic spectrum disorders. In all studies, ca ses and controls were unrela ted. GWAS summary statistic s for each cohort used the same methods a s for PG C29.

\section{Statistical methods}

We use GWAS summary statistic s and linka ge disequilbrium (D) score analysis (LDSC) (B. K. Bulik-Sullivan et al., 2015) to estimate the total proportion of va riance in lia bility attributa ble to SNPs genome-wide (i.e., SNP-herita bility). Bivariate LSC was used to estimate the genetic correlation tagged by genome-wide SNPs $\left(r_{g}\right)$ between two tra its. LDSC has been applied widely to GWAS summa ry statistic s of psychia tric (Anttila et al., 2018) and other disorders (B. Bulik-Sullivan et al., 2015), and results have been shown to agree well with estimates made

This article is protected by copyright. All rights reserved. 
from full individua l-level genotype and phenotype data using linear mixed model analysis (e.g., GREML (Yang et al., 2010)), a s long as the D reference sample is dra wn from a population that appropriately reflects the samples contributing the GWAS summary statistic s(Yang et al., 2015). A key advantage of LDSC is the minimal computational requirements compared to methods that use individual level data, and the ability to differentiate between genomic inflation due to polygenic ity and due to population stra tific a tion. Disadvanta ges of DSC a re that standard emors (s.e.) of estimates can be (about 50\%) higher compared to when estimates are based on full data, partic ula rly for $r_{g}$ estimates (Ni, Moser, Schizophrenia Working Group of the Psychiatric Genomics, Wray, \& Lee, 2018).

SNP-herita bility is estimated on the observed binary scale $h_{S N P-c c}^{2}$, but these estimates depend on the proportion of cases in the sample $(\mathrm{P})$ and so are not easily comparable ac ross cohorts. Hence, for improved interpreta bility and comparison across studies, $h_{S N P-c c}^{2}$ is transformed to the lia bility scale $h_{S N P}^{2}$ (Lee, Wray, Goddard, \& Visscher, 2011) based on normal distribution theory, given an assumed lifetime risk of disea se in the population(K):

$$
h_{S N P}^{2}=h_{S N P-c c}^{2} \frac{(K(1-K))^{2}}{P(1-P) z^{2}}
$$

where $z$ is the height of the standard nomal density function when truncated at proportion K. However, this transformation assumes that controls a re sc reened. Peyrot et al (2016) (Peyrot, Boomsma, Penninx, \& Wray, 2016) showed that when the proportion of controls that are unscreened is $u$, then transformation should be

$$
h_{S N P}^{2}=h_{S N P-c c}^{2} \frac{(K(1-K))^{2}}{P(1-P)(1-u K)^{2} Z^{2}}
$$

which reduces to equation [1] when all controls are screened, $u=0$. When diseases are uncommon, assuming controls a re screened when they are not makes little impact(Peyrot et

This article is protected by copyright. All rights reserved. 
al., 2016). However, for very common disorders, such as MDD, the difference is not trivial. For example, for $\mathrm{K}=0.15, h_{S N P-c c}^{2}=0.15, \mathrm{P}=0.5$, then $h_{S N P}^{2}=0.18$ when controls are screened and 0.24 when unscreened. The $r_{g}$ estimates a re robust to $P, K$ and $u$, since these factors contribute to both numerator and denominator of the correlation (which is defined as the estimate of the additive genetic covariance divided by the product of the square root of the SNP-herita bilities for the two tra its). Hence $r_{g}$ estimates a re robust to a sc erta inment practices and approximately the same where estimated on the case-control observed scale or liability scales (B. Bulik-Sullivan et al., 2015). If the sa me genetic effects contribute to disease risk between sexes or between cohorts then $r_{g}$ is expected to be 1 .

It was not possible to compare $h_{S N P}^{2}$ of each PGC29 cohort, because the per-cohort estimates had high s.e. (e.g. a cohort of 500 cases and 500 controls would be expected to produce $h_{S N P}^{2}$ with standard emor of at minimum 0.38 (Vissc her et al., 2014)). Instead we estimated the $h_{S N P}^{2}$ attributed to a cohort by evaluating its contribution to $h_{S N P}^{2}$ estimates calculated from 500 random samplings of cohorts drawn from the 29 PGC 29 cohorts. In each sampling, we randomly selected cohorts until the total sample size was $\geq 5000$, then used the GWAS summary statistic s meta-a nalysed (weighted by s.e.) in DSC to estimate $h_{S N P}^{2}$ assuming lifetime risk of $K=0.15$, and assuming controls are screened (equation [1]). To determine the contribution to the $h_{S N P}^{2}$ estimate from each cohort we fitted a linear model with estimated $h_{S N P}^{2}$ as the dependent variable regressed on indic ator va ria bles set as 1 if the cohort contributed to the estimate (was included in the random sampling), and 0 otherwise.

\section{RESULTS}

\section{Between-cohort heterogeneity within PGC29}

This article is protected by copyright. All rights reserved. 
We estimated $h_{S N P}^{2}$ in 500 random samplings of the cohorts from PGC29. From a linear regression of $h_{S N P}^{2}$ on ind ic a tor variables set as 1 if the cohort contributed to the estimate and 0 if it was not, we estimated an $h_{S N P}^{2}$ effect size deviation per cohort ( $\mathrm{y}$-axis Figure 1). Fifteen of the 29 cohorts had $h_{S N P}^{2}$ deviations different from zero $(p<0.05 / 29)$. We found that the cohorts nesl (combined sample of the Netherlands Study of Depression and Anxiety and the Netherlands Twin Registry) (Boomsma et al., 2008; Penninx et al., 2008) and gep3 (GenPod/NEWMEDS) (G. Lewis et al., 2011) contributed most to va riation in estimates of $h_{S N P}^{2}$, and explain 0.14 and 0.16 , respectively, of the variance in $h_{S N P}^{2}$ estimates across the 500 samplings. Samplings that included cohort nesl had the highest a verage estimates of $h_{S N P}^{2}$, while samplings including gep3 had the lowest average estimates. These differences are in line with expectations based on screening strategies for controls (Supplementary Table 1). The nesl cohort used super-screened controls (Boomsma et al., 2008), such that controls never scored higher than 0.65 on a general factor score for anxious depression (mean $=0$, $\mathrm{SD}=0.7$ ) derived from a combined measure of neurotic ism, anxiety, and depressive symptoms assessed via longitudinal questionnaires over 15 years. In contrast, the gep3 cohort was a case-only research cohort which was matched to independently collected and genotyped controls (hence particularly stringent $Q C$ is needed to combine the genotype data of the contributing cases and controls). In fact, gep3 is one of seven cohorts for which controls were unscreened for MDD (Figure 1), but only one other cohort used independently genotyped controls (STAR*D, coded as stm2); together the seven cohorts have lower mean beta-values, but not signific antly so $(p=0.055)$. The trend in these results might be explained by recognising that SNP-heritability is first estimated on the observed binary case-control scale $h_{S N P-c c}^{2}$ and then transformed to the liability scale $h_{S N P}^{2}$. Indeed, we find that increasing sample prevalence ( $\mathrm{P}$ in equation 1 ) is significantly associated with the estimated $h_{S N P}^{2}$ $(p=0.00057)$, but not sex ratio $(p=0.72)$. The application of the standard transfomation

This article is protected by copyright. All rights reserved. 
(equation [1]), as we have done, assumes screened controls and could generate an underestimate of the SNP-heritability if controls were in fact unsc reened. Similarly, super-sc reening of controls could generate an over-estimate of the true $h_{S N P}^{2}$. Hence, we expect that the standard transfomation would generate an overestimate for the nesl cohort (superscreened controls) and an underestimate for cohorts with unsc reened controls, consistent with our results.

Next, we investigated if $h_{S N P}^{2}$ estimates differed based on the research protocol to ascerta in cases. For the same proportion of cases and controls in the GWAS sample, we would expect the $h_{S N P-c c}^{2}$ to be higher for a c linic a lly a scerta ined cohort than a community ascerta ined cohort, further we would expect the transformation based on $\mathrm{K}=0.15$ (equation [1]) to overestimate $h_{S N P}^{2}$ when the true $\mathrm{K}$ is lower (clinical cohort) and underestimate $h_{S N P}^{2}$ when the true $\mathrm{K}$ is higher (community cohort). There is evidence to support this hypothesis (Figure 1). We found significant difference between the mean estimates of community $(-0.027$, s.e. 0.007 ) vs non-community cohorts (-0.08 s.e. 0.006$)$ (with non-community comprising the three in- and out-patient c ategories), using a one-sided, two-sa mple t-test assuming unequal variance $(p=0.028)$ (Supplementary Table 4$)$. The difference became more signific ant $(p=0.015)$ when the cohorts we had a priori reason to exclude, namely nesl and gep3, based on discussions above were removed.

\section{Between-sex heterogeneity}

Using the fourlarge data sets (Table 1) we investigate sex-specific heterogeneity. We used bivariate LSC to estimate the $r_{g}$ between all pairs of the two sexes by four data sets, but the standard emors were high (Supplementary Table 2). $r_{\text {g }}$ involving the GERA_M data set were not estimable, because of the negative/zero of $h_{S N P}^{2}$ used in the denominator of the $\mathrm{r}_{\mathrm{g}}$

This article is protected by copyright. All rights reserved. 
estima te. The meta-a na lysis of 12 ma le-fema le $r_{g}$ estima tes was 0.74 (s.e. $0.043 ; p_{\text {Ho:rg }=1}=1.9$ $\left.\times 10^{-9}\right)$. At face value this estimate implies genetic factors are only partially sha red between the sexes. However, this interpretation should be considered with caution when benchmarked by the meta-a na lysis of 6 female-female $r_{g}$ estima tes of 0.71 (s.e. 0.051 ; $\left.p_{\text {H0:rg }=1}=1.0 \times 10^{-8}\right)$ and the meta-analysis of 3 male-male $r_{g}$ estimates of 0.75 (s.e. $0.13 ; p_{\text {H0:rg }=1}=$ 0.05) Hence, the between-sex estimate of $r_{g}$ being signific antly different from zero likely reflects the general heterogeneity between the data sets rather than being sex-specific . Indeed, the between-sex $r_{g}$ estimated from the meta-a nalysis of the GWAS summary statistic $S$

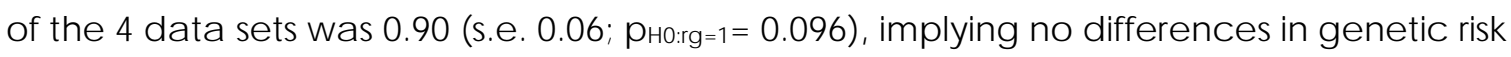
factors between the sexes.

Next, we investigated sex-specific estima tes of $h_{S N P}^{2}$ using LSC (Table 2, Supplementary Table 3) to determine if there is evidence for a greater genetic contribution to MDD risk in females then males. We have power to detect differences of the order of $2^{*}$ (s.e. of male estimate + s.e. of fema le estimate). Initially, in the transformation of the $h_{S N P-c c}^{2}$ estimate to the liability scale (equation [1]) we assumed $K=0.20$ for females and $K=0.10$ for males (Table 2), consistent with literature reports that MDD is twice as common in females as males (Weissman, Leaf, Holzer, Myers, \& Tisc hler, 1984). The $h_{S N P}^{2}$ estima tes were smaller for males (range -0.02 to 0.15 ) than for females (range 0.15 to 0.23 ), but given the magnitude of the standard emors, none of the $h_{S N P}^{2}$ sex differences were signific a ntly different for any individual data set. Meta-analysis of the $h_{S N P}^{2}$ estimates of the four data sets did lead to estimates that were signific antly different (Meta-4 in Table 2; 0.12 in ma les vs. 0.19 in females, $p=0.0016$ ). However, $h_{S N P}^{2}$ estimated from the meta-a nalysed GWAS results of the 4 data sets showed only nominal signific ance for the difference between males and females ( 0.10 vs $0.12, p=$ 0.041; Table 2 GWAS-Meta). This disc repancy can be expla ined, in part, by the fact that the

This article is protected by copyright. All rights reserved. 
meta-a nalysed GWAS summary statistic s inc lude assoc iation signal from between data-sets as well as within-data sets, and/or may be diluted by between data set heterogeneity. To investigate this discrepancy, we also meta-analysed the six $h_{S N P}^{2}$ values estimated from the genetic covariance between pairs of same-sex data sets in bivariate LDSC a nalysis. Since the traits are (presumed to be) the same, the genetic covariance is also an estimate of genetic va riance (Supplementary Table 3; Table $\mathbf{2}$ Meta-6). This a ga in showed lower mean estimates for males with a signific ant difference between the sexes (0.09 in males vs 0.12 in females, $p=0.0070$ ). For completeness, a meta-a na lysis from all 10 of the estimates is provided (Table 2 Meta-10); this uses the same data sets as the GWAS-Meta, but the latter uses all the information jointly rather than pairwise.

For completeness, we also estimated X-chromosome SNP-herita bility from the meta-analysed cohorts formales and females separately. However, the standard errors of the estimates were large relative to the $h_{S N P}^{2}$ estimates $\left(h_{S N P}^{2}\right.$ males $=0.0025(\mathrm{se}=0.06) ; h_{S N P}^{2}$ females $=0.0005$ (se $=0.03)$, which meant estimation of the $r_{g}$ between them was not meaningful.

Before drawing strong conclusions from these results, it is important to recognise that the estimates of $h_{S N P}^{2}$ depend on the choice of the lifetime risk estimates ( $K$ in equations [1] and [2]) (Figure 2). The point estimates a re more similar if the sa me lifetime risk is assumed between the sexes, but it is diffic ult to justify such an assumption, because it is not, at face value, supported by epidemiological data. However, since depression maybe underreported in males (Martin, Neighbors, \& Griffith, 2013; Tho mic roft et al., 2017), for illustration purposes we could assume the true lifetime risk of MDD is the same between the sexes (K $=0.20$ ), but that through under-reporting the controls a re conta minated by 0.10 of cases

This article is protected by copyright. All rights reserved. 
(Equation [2], $\mathrm{u}=0.1$ ). Under these assumptions, the $h_{S N P}^{2}$ estima tes a re not signific a ntly different between the sexes for any data set (Figure 2, Table 2)

\section{DISCUSSION}

Heterogeneity in MDD is often disc ussed, but hard to investigate. In a novel set of analyses, we explored the heterogeneity of MDD using genetic data. The first set of analyses contrasted 29 PGC cohorts, by estimating their a verage contribution to estimates of $h_{S N P}^{2}$ from repeated random samplings of cohorts selected into GWAS meta-a nalyses. While we found notable differences between cohorts in the $h_{S N P}^{2}$ contribution estimates (Figure 1), these differences could be explained, at least partly, via knowledge of cohort ascerta inment practices: higher contributions for c ohorts ascerta ined in clinical compared to community settings (Figure 1, $p=0.028$ ), higher contribution from a sample known to use super-sc reened controls (nes1), and a trend towards lower contributions from samples that used unsc reened controls. One conclusion is that known cohort information about case asc erta inment status could be included usefully in a nalysis methods to increase power. A framework for such an a na lysis has been proposed (Zaitlen et al., 2012), but in practice the necessary parameters relating to cohort specific risks a re usually unknown. In the seven samples contributing to the published PGC meta-a nalysis (PGC29, GERA, iPSYCH, UK Biobank, deC ode, Generation Scotland, 23andMe) (Wray et al., 2018), $h_{S N P}^{2}$ estimates ranged from 0.09 to 0.25 and the weighted mean $r_{g}$ for all pa inwise combinations was $0.76($ s.e. $=0.03)$, whic $h$ is signific antly different from one. The cohorts had different recruitment strategies with ascerta inment ranging from self-report to national hospital records. Moreover, even within the wave 1 PGCMDD research cohorts endorsement proportions of the nine DSMIV criteria showed considerable heterogeneity including between cohorts that had similar c linical ascerta inment strategies(Major Depressive Disorder Working Group of the Psychiatric et al.,

This article is protected by copyright. All rights reserved. 
2013). For example, endorsement rates of $56 \%, 27 \%$ and $10 \%$ were recorded for the criterion symptom $4 b$, hypersomnia nearly every day, for different early onset ( $<30$ years) recurrent MDD samples(Major Depressive Disorder Working Group of the Psychiatric et al., 2013). Despite the heterogeneity, out-of-sample prediction demonstrated that the self-reported 23andMe GWAS results expla ined variance in clinic ally ascerta ined cohorts with high signific ance (Wray et al., 2018). Sample size remains the driving force for genetic discovery in MDD. Ideally, la rger sample sizes should be accompanied by collection of detailed, consistent, and longitudinal phenotypic data to enable more precise case and control definitions.

We also investigated between-sex genetic heterogeneity. Our sex-specific analyses found signific antly smaller $h_{S N P}^{2}$ for ma les than females, a trend replic ated in all fourdata sets, and hence was highly signific ant in the meta-a na lysis of the four cohort estima tes (Table $\mathbf{2}$ Meta4). However, a set of follow-up a nalyses leads to a somewhat different conclusion. Since the $h_{S N P}^{2}$ estimates generated from the meta-analysis of the summary statistic s of the four data sets was only nomina lly signific a ntly different (Table 2, G WAS-Meta, ma les vs. females: 0.10 s.e. 0.01 vs. 0.12 s.e. $0.01, p=0.041$ ), it could be argued that heterogeneity between data sets (as reported in our between cohort a nalyses above) removes a signal in sex differences, a point of view supported by the lower $h_{S N P}^{2}$ estimates for both sexes. However, it is noteworthy that for the largest sample, with data collected under standardised protocols (UKB), the $h_{S N P}^{2}$ point estimates were similar (Table 2). Moreover, we recognised that the comparisons of $h_{S N P}^{2}$ between the sexes depended on the choic e of their respective lifetime risks (Figure 2). For ba seline a na lyses we used lifetime risk estima tes of $K=0.20$ for fema les and $K=0.10$ for males, consistent with a 2:1 risk for fema les vs. ma les (Weissman et al., 1984), with higher K values generating higher $h_{S N P}^{2}$ estimates. One expla nation for a lower lifetime risk for males

This article is protected by copyright. All rights reserved. 
could be higher rates of under-reporting (Martin et al., 2013; Thomic roft et al., 2017). We calculated $h_{S N P}^{2}$ in males assuming the same lifetime risk as females, but with incomplete sc reening of controls. Such a hypothetic al sc enario generated similar estimates of $h_{S N P}^{2}$ between the sexes (Figure 2, Table 2). The last piece of evidence supporting no sex differences in genetic factors contributing to depression is the high estimated $r_{g}$ between the sexes ( 0.90 s.e. $\left.0.06 ; \mathrm{p}_{\text {HO: } \mathrm{rg}=1}=0.096\right)$.

In summary, our a nalyses demonstrate between-cohort genetic heterogeneity, but this can be explained, at least in part, by known factors such as case/control ascerta inment. Investigation of between sex heterogeneity provided no convinc ing evidence to support genetic differences between the sexes. A robust conclusion is simply that la rge sample sizes will overcome sample heterogeneity as demonstrated in the latest major depression GWAS meta-analyses (Howard et al., 2018; Wray et al., 2018). Ba sed on differences in lifetime disea se risk and differences in herita bility, while assuming a similar number of contributing risk loci, we previously estimated that sample sizes for GWAS need to be five times bigger for MDD than for sc hizophrenia (SCZ) (Wray et al., 2012). On the one hand, heterogeneity between samples may push this estimate higher. On the other hand, the heterogeneity may a lready account for the higher prevalence and lower herita bility. The PGC GWAS metaa na lysis for MDD/major depression based on 135K c ases (Wray et al., 2018) identified 44 independent signific ant loci. This compares to 145 independent loci for SC Z from 41K cases (Pa rdiñas et al., 2018), hence requining 3.4 times as many cases for major depression compared to SCZpergenome-wide significant loc us. However, the relationship between sample size and variant disc overy is not linear (Wray et al., 2018) and so observing the sample size ratios for disc overy will be of interest as sample sizes increase. Very large MDD case-control samples will a llow novel methods to be applied to assess evidence for genetic

This article is protected by copyright. All rights reserved. 
subsets. Larger data sets a re likely to lead to the development of new methods to assess genetic heterogeneity (Han et al., 2016). There is a growing interest in machine lea ming methods (Libbrecht \& Noble, 2015) as a stra tegy to identify phenotypically relevant genetic subsets, but cohort heterogeneity must diminish their utility, making large electronic health or biobank samples collected and genotyped in a uniform way of most value.

\title{
Acknowledgements
}

We acknowledge funding from the Australian National Health \& Medical Research Council (1078901, 1113400, 1087889). The PGC has received major fund ing from the US National Institute of Mental Health and the US National Institute of Drug Abuse (U01 MH109528 and U01 $\mathrm{MH1095320).} \mathrm{A} \mathrm{full} \mathrm{list} \mathrm{of} \mathrm{fund} \mathrm{ing} \mathrm{is} \mathrm{provided} \mathrm{in} \mathrm{ref(Wray} \mathrm{et} \mathrm{al.,} \mathrm{2018).} \mathrm{UK} \mathrm{Biobank:} \mathrm{this} \mathrm{research}$ has been conducted using the UK Biobank 593 Resource (URLS), including applic ations \#4844 and \#6818. The Genetic Epidemiology Research on Adult Health and Aging (GERA) study was supported by grant RC2 AG 036607 from the National Institute of Health, grants from Robert Wood J ohnson Foundation, the Ellison Medic al Foundation, the Wayne and Gladys Valley Foundation and Ka iser Permanente. The a uthors thank the Kaiser Permanente Medic al Care Plan, Northem Califomia Region (KPNC) members who have generously agreed to partic ipate in the Ka iser Permanente Research Program on Genes, Environment and Health (RPGEH).

\author{
Author Contributions \\ Study design: MT, DM, NGM, DFS, NRW; Structured methodological review of PGC Cohorts: \\ KSK, DFS, Analysis: MT, DM,DH, RM Analysis-PGC: SR, Analysis-UKB: DD,DH,KEK,J S, MT, AMc \\ Analysis-Xchromosome: J S, MT. Analysis-iPSYCH: MM,ADB. PGC-MDD Principal Investigators: \\ $B T B, H J G, A C H, P A F M$ PGC-MDD Steening Committee: PFS, CML, G B, ADB, DFL, NRW. First draft \\ of manuscript MT, NRW. Final manusc ript all a uthors
}

This article is protected by copyright. All rights reserved. 
Table 1. Description of GWAS data sets for between-sex heterogeneity analyses

\begin{tabular}{|l|r|r|r|r|r|r|r|}
\hline Data Set & \multicolumn{1}{|l|}{ Cases } & \multicolumn{1}{|l|}{ Controls } & \multicolumn{1}{l}{$\begin{array}{l}\text { Female } \\
\text { cases }\end{array}$} & $\begin{array}{l}\text { Female } \\
\text { controls }\end{array}$ & $\begin{array}{l}\text { Male } \\
\text { cases }\end{array}$ & $\begin{array}{l}\text { Male } \\
\text { controls }\end{array}$ & $\begin{array}{l}\text { Number } \\
\text { of } \\
\text { Cohortsa }\end{array}$ \\
\hline PGC29 & 16,823 & 25,632 & 11,438 & 12,463 & 5,377 & 13,022 & $29 \mathrm{~b}$ \\
\hline GERA & 7,162 & 38,287 & 5,152 & 20,650 & 2,010 & 17,637 & 1 \\
\hline UKB & 113,769 & 208,801 & 73,292 & 99,385 & 40,477 & 109,426 & 1 \\
\hline iPSYCH & 18,577 & 17,637 & 12,690 & 8,534 & 5,887 & 9,103 & 1 \\
\hline Total & $\mathbf{1 5 6 , 3 3 1}$ & $\mathbf{2 9 0 , 3 5 7}$ & $\mathbf{1 0 2 , 5 7 2}$ & $\mathbf{1 4 1 , 0 3 2}$ & $\mathbf{5 3 , 7 5 1}$ & $\mathbf{1 4 9 , 1 8 8}$ & $\mathbf{3 2}$ \\
\hline
\end{tabular}

a: Cohort is defined as the cases and controls with genome-wide genotypes imputed from the same set of SNPs that have passed through a common quality control pipeline. Mostly, cohort reflects a case-control sample collected by a PGC principal investigator. b: cohorts ranged in size from 246 to 3760 cases plus controls.

This article is protected by copyright. All rights reserved. 
Table 2 Estimates of $h_{S N P}^{2}$ from LSC applied to sex-spec ific GWAS summary statistics

\begin{tabular}{|c|c|c|c|c|c|}
\hline & Female (se) & Males v1 (se) & Males v2 (se) & $\begin{array}{l}\text { P-value } \\
\text { v1 }\end{array}$ & \begin{tabular}{|l} 
P-value \\
v2
\end{tabular} \\
\hline K & 0.2 & 0.1 & 0.2 & & \\
\hline $\mathbf{u}$ & $\mathbf{0}$ & $\mathbf{0}$ & 0.1 & & \\
\hline PGC29 & $0.20 \quad(0.03)$ & $0.07 \quad(0.04)$ & $0.09 \quad(0.05)$ & 0.60 & 0.68 \\
\hline GERA & $0.15 \quad(0.04)$ & $-0.02 \quad(0.05)$ & $-0.03 \quad(0.07)$ & 0.55 & 0.57 \\
\hline UKB & $0.18 \quad(0.02)$ & $0.15 \quad(0.02)$ & $0.20 \quad(0.03)$ & 0.90 & 0.93 \\
\hline iPSYCH & $0.23 \quad(0.03)$ & $0.15 \quad(0.04)$ & $0.20 \quad(0.05)$ & 0.77 & 0.91 \\
\hline Meta-4 & $0.19 \quad(0.01)$ & $0.12 \quad(0.02)$ & $0.16 \quad(0.02)$ & $1.6 \times 10^{-3}$ & 0.23 \\
\hline Meta-6 & $0.12 \quad(0.01)$ & $0.09 \quad(0.01)$ & $0.11 \quad(0.01)$ & $7.0 \times 10^{-3}$ & 0.58 \\
\hline Meta-10 & $0.14 \quad(0.01)$ & $0.10 \quad(0.01)$ & $0.12 \quad(0.01)$ & $8.6 \times 10^{-5}$ & 0.28 \\
\hline GWAS-Meta & $0.12 \quad(0.01)$ & $0.10 \quad(0.01)$ & $0.12 \quad(0.01)$ & $4.1 \times 10^{-2}$ & 0.92 \\
\hline
\end{tabular}

$h_{S N P}^{2}$ estimates are presented on the lia bility scale achieved through transformation of the LDSC $h_{S N P-c c}^{2}$ estimate accounting for the case prevalence in the sample (P), the lifetime risk $(K)$ of the disorder, and the proportion of cases in the control sample (u), equation [1]. Meta4: meta-a nalysis of the $h_{S N P}^{2}$ estimates for the 4 data sets (PGC 29,GERA,UKB, iPSYCH). Meta-6: meta-analysis of the $6 h_{S N P}^{2}$ estimates derived from the genetic covariance estimates from bivariate DSC between the 6 possible same-sex data-set pa irwise combinations. Meta-10: meta-a na lysis based on all $h_{S N P}^{2}$ estimates contributing to Meta-4 and Meta-6. GWAS-Meta: $h_{S N P}^{2}$ estimated from the GWAS summary statistic s of the 4 data sets. Versions v1 and v2 differ by K and u values; $v 2$ hypothesis that the lifetime risk of MDD is the same in men and women but that more cases go unreported in men and hence could be included in a screened control set.

This article is protected by copyright. All rights reserved. 
Figure 1. Cohort deviation estimates from the linear regression of $h_{S N P}^{2}$ estimates (from each of the $\mathbf{5 0 0}$ samplings of cohorts) on cohort indic ator variables set at 1 if the cohort was included in the sampling that generated the $h_{S N P}^{2}$ and 0 othenwise.

In each sampling, cohorts were selected at random until the total case/control sample size exceeded 5000. Cohort GWAS results were meta-analysed and these results passed into Dscore. $h_{S N P}^{2}$ was estimated using the equation 1 transfomation $(\mathrm{K}=0.15)$ which assumes controls a re screened. $h_{S N P}^{2}$ estimates of samplings were highest, on a verage, when cohort nesl was included and lowest, on average, when cohort gep3 was included. Wave 1 cohorts have an asterisk by their name and cohorts that have unscreened controls are marked by a tilde. Continuous lines around data-points are $95 \%$ confidence Intervals. For explanation of cohort names see Supplementary Table 1.

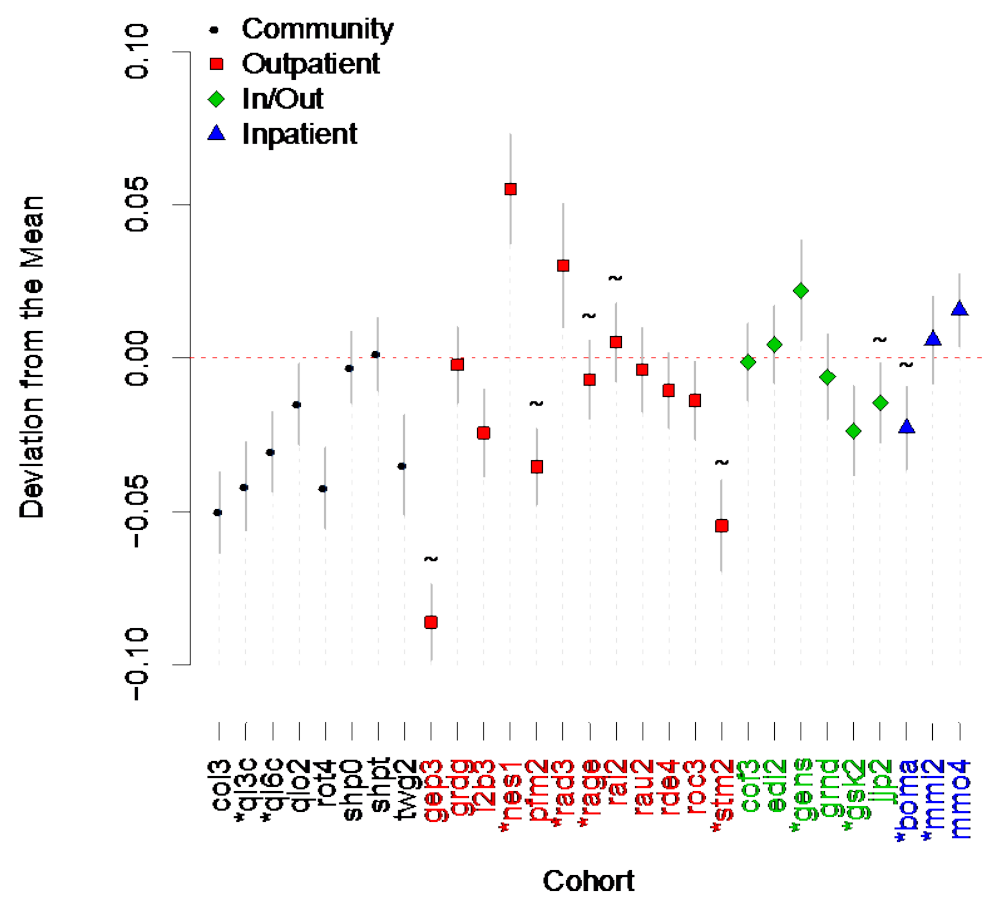

This article is protected by copyright. All rights reserved. 
Figure 2. Impact of choice of lifetime risk on estimate of $\boldsymbol{h}_{S N P}^{2}$. The graphs shows $h_{S N P}^{2}$ on the liability scale from equation [2], u (proportion of controls that are unrecognised cases). The blue/red dashed lines are positioned at the lifetime risk formales/females. The flat ended bars show the $95 \%$ confidence intervals of the $h_{S N P}^{2}$ estimates at the chosen lifetime risk.

PGC29

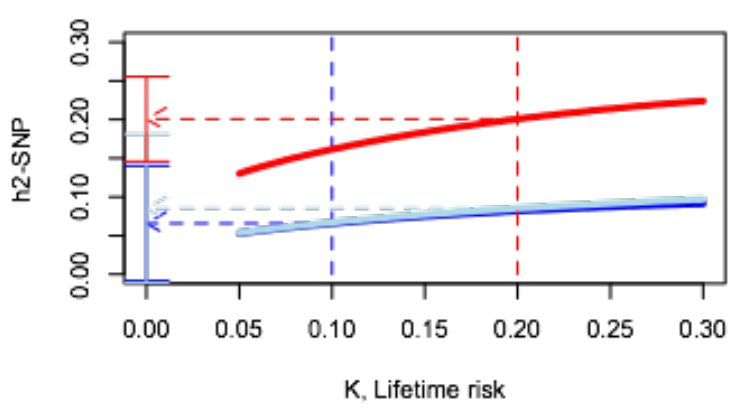

UKB

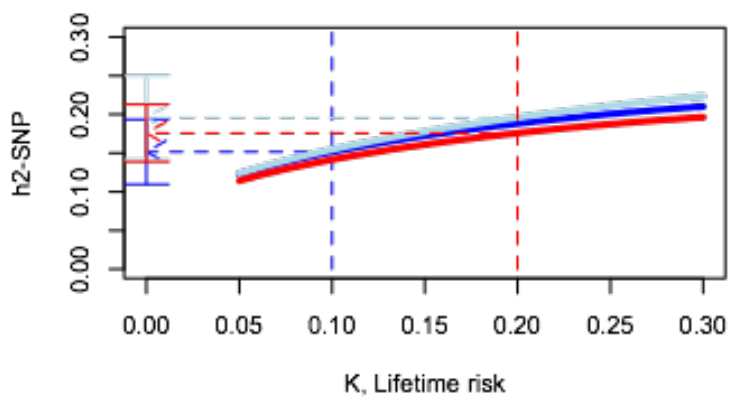

GWAS-Meta

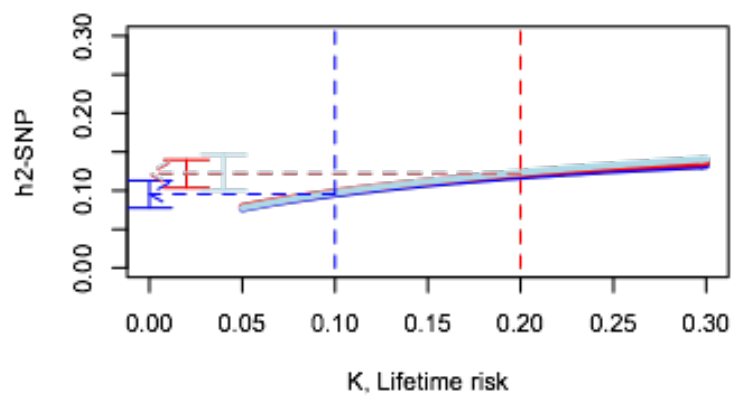

GERA

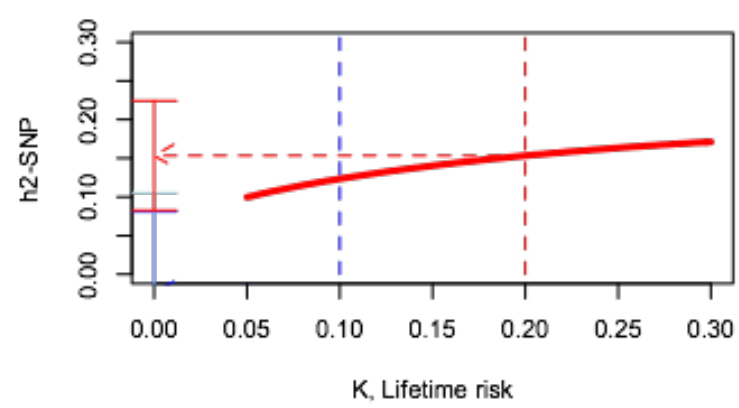

iPSYCH

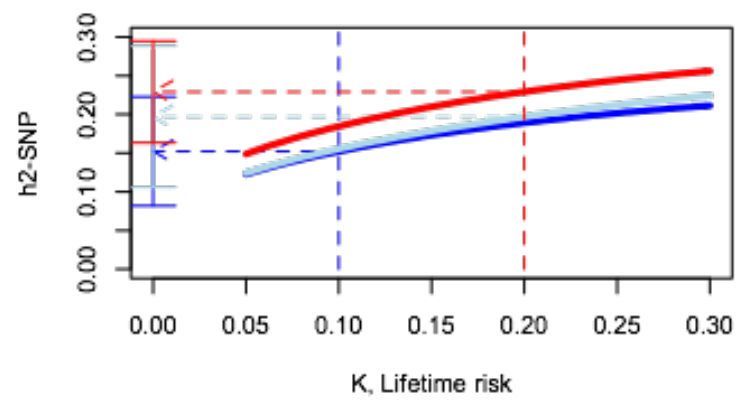

Male, $u=0.1$

Female, $u=0$

Male, $u=0$

This article is protected by copyright. All rights reserved. 


\section{References}

American Psychiatric Association. (1994). Diagnostic and Statistic al Manual of Mental Disorders (Fourth Edition ed.). Washington, DC: American Psychiatric Association.

Anttila, V., Bulik-Sullivan, B., Finuca ne, H. K., Walters, R. K., Bras, J ., Duncan, L., ... Neale, B. M. (2018). Ana lysis of shared herita bility in common disorders of the brain. Science, 360(6395).

Banda, Y., Kvale, M. N., Hoffmann, T. J ., Hesselson, S. E., Ranatunga, D., Tang, H., ... Risch, N. (2015). Characterizing Race/Ethnic ity and Genetic Ancestry for 100,000 Subjects in the Genetic Epidemiology Research on Adult Health and Aging (GERA) Cohort. Genetics, 200(4), 1285-1295. doi:10.1534/genetic s.115.178616

Boomsma, D. I., Willemsen, G., Sullivan, P. F., Heutink, P., Meijer, P., Sondervan, D., . . Penninx, B. W. (2008). Genome-wide association of major depression: description of samples for the GAIN Major Depressive Disorder Study: NTR and NESDA biobank projects. Eur J Hum Genet, 16(3), 335-342. doi:10.1038/sj.ejhg.5201979

Bruce, M. L., \& Hoff, R. A. (1994). Soc ial a nd physic al hea th risk factors for first-onset major depressive disorder in a community sample. Social Psychiatry and Psychiatric Epidemiology, 29(4), 165-171. doi:10.1007/BF00802013

Bulik-Sullivan, B., Finuca ne, H. K., Anttila, V., Gusev, A., Day, F. R., Loh, P. R., . . Neale, B. M. (2015). An atlas of genetic correla tions a cross human disea ses and tra its. Nat Genet, 47(11), 1236-1241. doi:10.1038/ng.3406

Bulik-Sullivan, B. K., Loh, P. R., Finuc ane, H. K., Ripke, S., Yang, J., Sc hizophrenia Working Group of the Psychiatric Genomic s, C., ... Neale, B. M. (2015). L Score regression distinguishes confounding from polygenic ity in genome-wide association studies. Nat Genet, 47(3), 291-295. doi:10.1038/ng.3211

Bycroft, C., Freeman, C., Petkova, D., Band, G ., Elliott, L. T., Sharp, K., ... Marchini, J . (2018). The UK Biobank resource with deep phenotyping and genomic data. Nature, 562(7726), 203-209. doi:10.1038/s41586-018-0579-z

Chapman, D. P., Whitfield, C. L., Felitti, V. J ., Dube, S. R., Edwa rds, V. J ., \& Anda, R. F. (2004). Adverse child hood experiences and the risk of depressive disorders in a dulthood. J Affect Disord, 82(2), 217-225. doi:10.1016/j.ja d.2003.12.013

Cross-Disorder Group of the Psychiatric Genomics, C., Lee, S. H., Ripke, S., Neale, B. M., Fa ra one, S. V., Purc ell, S. M., ... Intemational Infla mmatory Bowel Disea se Genetics, C. (2013). Genetic relationship between five psychiatric disorders estimated from genome-wide SNPs. Nat Genet, 45(9), 984-994. doi:10.1038/ng.2711

Fanous, A. H., \& Kendler, K. S. (2005). Genetic heterogeneity, modifier genes, and quantitative phenotypes in psychiatric illness: searching for a framework. Mol Psychiatry, 10(1), 6-13. doi:10.1038/sj.mp.4001571

Femandez-Pujals, A. M., Adams, M. J ., Thomson, P., McKechanie, A. G., Blackwood, D. H., Smith, B. H., ... Mc Intosh, A. M. (2015). Epidemiology and Herita bility of Major Depressive Disorder, Stratified by Age of Onset, Sex, and Illness Course in Generation Sc otland: Scottish Family Health Study (G S:SFHS). PLoS One, 10(11), e0142197. doi:10.1371/joumal.pone.0142197

Fiedorowic Z, J. G. (2014). Depression and Cardiova sc ular Disea se: An Update on How Course of Illness May Influence Risk. Current psyc hiatry reports, 16(10), 492-492. doi:10.1007/s11920-014-0492-6

Fraguas J r, R., losifesc u, D. V., Alpert, J ., Wisniewski, S. R., Barkin, J . L., Trivedi, M. H., . . Fava, M. (2007). Major Depressive Disorder and Comorbid Cardiac Disea se: Is There a

This article is protected by copyright. All rights reserved. 
Depressive Subtype With Greater Card iovascular Morbidity? Results From the STAR*D Study. Psychosomatics, 48(5), 418-425. doi:doi:10.1176/a ppi.psy.48.5.418

Genomes Project, C., Abecasis, G. R., Altshuler, D., Auton, A., Brooks, L. D., Durbin, R. M., ... McVean, G. A. (2010). A map of human genome variation from population-scale sequencing. Nature, 467(7319), 1061-1073. doi:10.1038/nature09534

Han, B., Pouget, J. G., Slowikowski, K., Sta hl, E., Lee, C. H., Diogo, D., . . . Ra yc ha ud huri, S. (2016). A method to decipher pleiotropy by detecting underlying heterogeneity driven by hidden subgroups a pplied to autoimmune and neuropsychiatric diseases. Nat G enet, 48(7), 803-810. doi:10.1038/ng.3572

Heim, C., Newport, D. J ., Mletzko, T., Miller, A. H., \& Nemeroff, C. B. (2008). The link between child hood tra uma and depression: Insights from HPA axis studies in humans. Psychoneuroendoc rinology, 33(6), 693-710. doi:doi:10.1016/j.psyneuen.2008.03.008

Howard, D. M., Adams, M. J ., Shirali, M., Clarke, T.-K., Marioni, R. E., Davies, G., . . Mc Intosh, A. M. (2018). G enome-wide assoc iation study of depression phenotypes in UK Biobank identifies variants in excitatory syna ptic pathways. Nature Communications, 9(1), 1470. doi:10.1038/s41467-018-03819-3

Huffman, J. C., Celano, C. M., Beach, S. R., Motiwa la, S. R., \& J a nuzzi, J . L. (2013). Depression and cardiac disease: epidemiology, mechanisms, and diagnosis. Cardiovasc Psyc hiatry Neurol, 2013, 695925. doi:10.1155/2013/695925

Hunt, M., Auriemma, J ., \& Cashaw, A. C. (2003). Self-report bias and underreporting of depression on the BDI-II. J Pers Assess, 80(1), 26-30. doi:10.1207/s15327752jpa8001_10

Kapur, S., Phillips, A. G., \& Insel, T. R. (2012). Why has it ta ken so long for biologic al psychiatry to develop clinic al tests and what to do about it? Mol Psychiatry, 17(12), 1174-1179. doi:10.1038/mp.2012.105

Kendler, K. S., Gatz, M., Gardner, C. O., \& Pedersen, N. L. (2006). A Swedish national twin study of lifetime major depression. Am J Psychiatry, 163(1), 109-114. doi:10.1176/appi.ajp.163.1.109

Kessler, R. C. (2003). Epid emiology of women and depression. J oumal of Affective Disorders, 74(1), 5-13. doi:doi:10.1016/S0165-0327(02)00426-3

Kessler, R. C., \& Bromet, E. J. (2013). The epidemiology of depression ac ross cultures. Annu Rev Public Health, 34, 119-138. doi:10.1146/a nnurev-publhealth-031912-114409

Lane, J. M., Vlasac, I., Anderson, S. G., Kyle, S. D., Dixon, W. G., Bechtold, D. A., . . Saxena, R. (2016). Genome-wide association a nalysis identifies novel loci for chronotype in 100,420 individua Is from the UK Biobank. Nat Commun, 7, 10889. doi:10.1038/ncomms10889

Lee, S. H., Wray, N. R., Goddard, M. E., \& Visscher, P. M. (2011). Estimating missing herita bility for disease from genome-wide assoc iation studies. Am J Hum Genet, 88(3), 294-305. doi:10.1016/j.ajhg.2011.02.002

Lewis, C. M., Ng, M. Y., Butler, A. W., Cohen-Woods, S., Uher, R., Pirlo, K., . . McG uffin, P. (2010). Genome-wide association study of major recurrent depression in the U.K. population. Am J Psychiatry, 167(8), 949-957. doi:10.1176/appi.ajp.2010.09091380

Lewis, G., Mulligan, J., Wiles, N., Cowen, P., Craddock, N., Ikeda, M., . . Peters, T. J . (2011). Polymorphism of the 5-HTtransporter and response to antidepressants: randomised controlled trial. BrJ Psychiatry, 198(6), 464-471. doi:10.1192/bjp.bp.110.082727

Libbrecht, M. W., \& Noble, W. S. (2015). Machine leaming a pplications in genetic s and genomics. Nat Rev Genet, 16(6), 321-332. doi:10.1038/nrg3920

Lohoff, F. W. (2010). Overview of the Genetic s of Major Depressive Disorder. Current psychiatry reports, 12(6), 539-546. doi:10.1007/s11920-010-0150-6

Major Depressive Disorder Working Group of the Psychiatric, G. C., Ripke, S., Wray, N. R., Lewis, C. M., Ha milton, S. P., Weissman, M. M., . . Sullivan, P. F. (2013). A mega-

This article is protected by copyright. All rights reserved. 
a nalysis of genome-wide association studies for major depressive disorder. Mol Psychiatry, 18(4), 497-511. doi:10.1038/mp.2012.21

Martin, L. A., Neighbors, H. W., \& G riffith, D. M. (2013). The experience of symptoms of depression in men vs women: a nalysis of the National Comorbid ity Survey Replication. J AMA Psychiatry, 70(10), 1100-1106. doi:10.1001/ja ma psychia try.2013.1985

McGuffin, P., Katz, R., Watkins, S., \& Rutherford, J . (1996). A hospita l-based twin register of the herita bility of DSM-IV unipolar depression. Arch G en Psychiatry, 53, 129-136.

Mosing, M. A., Gordon, S. D., Medland, S. E., Statham, D. J ., Nelson, E. C., Heath, A. C., ... Wray, N. R. (2009). Genetic and environmental influences on the co-morbidity between depression, panic disorder, agoraphobia, and social phobia: a twin study. Depress Anxiety, 26(11), 1004-1011. doi:10.1002/da.20611

Ni, G., Moser, G., Schizophrenia Working Group of the Psychiatric Genomic s, C., Wray, N. R., $\&$ Lee, S. H. (2018). Estimation of Genetic Correlation via Linkage Disequilibrium Score Regression and Genomic Restricted Maximum Likelihood. Americ an joumal of human genetic s, 102(6), 1185-1194. doi:10.1016/j.ajhg.2018.03.021

Pardiñas, A. F., Holmans, P., Poc klington, A. J ., Esc ott-Price, V., Ripke, S., Carrera, N., . . . Walters, J. T. R. (2018). Common sc hizophrenia alleles are enriched in mutationintolerant genes and in regions under strong background selection. Nature Genetic s, 50(3), 381-389. doi:10.1038/s41588-018-0059-2

Penninx, B. W., Beekman, A. T., Smit, J. H., Ztman, F. G., Nolen, W. A., Spinhoven, P., . . Consortium, N. R. (2008). The Netherlands Study of Depression and Anxiety (NESDA): rationale, objectives and methods. Int J Methods Psychiatr Res, 17(3), 121-140. doi:10.1002/mpr.256

Peyrot, W. J ., Boomsma, D. I., Penninx, B. W., \& Wray, N. R. (2016). Disea se and Polygenic Architecture: Avoid Trio Design and Appropriately Account for Unsc reened Control Subjects for Common Disea se. Am J Hum Genet, 98(2), 382-391. doi:10.1016/j.ajhg.2015.12.017

Schuch, J. J ., Roest, A. M., Nolen, W. A., Penninx, B. W., \& de J onge, P. (2014). Gender differences in major depressive disorder. results from the Netherlands study of depression and anxiety. J Affect Disord, 156, 156-163. doi:10.1016/j.jad.2013.12.011

Sullivan, P. F., Neale, M. C., \& Kendler, K. S. (2000). Genetic epidemiology of major depression: review and meta-analysis. Am J Psychiatry, 157(10), 1552-1562. doi:10.1176/appi.a.jp.157.10.1552

Thomic roft, G., Chatterji, S., Evans-Lacko, S., Gruber, M., Sampson, N., Aguila r-Gaxiola, S., ... Kessler, R. C. (2017). Undertreatment of people with major depressive disorder in 21 countries. BrJ Psychiatry, 210(2), 119-124. doi:10.1192/bjp.bp.116.188078

Visscher, P. M., Hemani, G., Vinkhuyzen, A. A. E., Chen, G. B., Lee, S. H., Wray, N. R., . . Yang, J. (2014). Statistic al Power to Detect Genetic (Co)Variance of Complex Tra its Using SNP Data in Unrelated Samples. Plos Genetics, 10(4). doi:ARTN e1004269

10.1371/joumal.pgen.1004269

Vythilingam, M., Heim, C., Newport, J ., Miller, A. H., Anderson, E., Bronen, R., . . Bremner, J . D. (2002). Childhood trauma associated with smaller hippocampal volume in women with major depression. Am J Psychiatry, 159(12), 2072-2080. doi:10.1176/appi.ajp.159.12.2072

Weissman, M. M., Leaf, P. J ., Holzer, C. E., 3rd, Myers, J . K., \& Tisc hler, G . L. (1984). The epidemiology of depression. An update on sex differences in rates. J Affect Disord, 7(3-4), 179-188.

World Health Organization. (1978). Intemational Classific ation of Diseases (9th revised ed.). Geneva: World Health Organization.

This article is protected by copyright. All rights reserved. 
World Health Organization. (1992). Intemational Classific ation of Disea ses (10th revised ed.). Geneva: World Health Organization.

Wray, N. R., Perga dia, M. L., Bla ckwood, D. H., Penninx, B. W., Gordon, S. D., Nyholt, D. R., . . Sullivan, P. F. (2012). Genome-wide association study of major depressive disorder: new results, meta-a nalysis, and lessons leamed. Mol Psyc hiatry, 17(1), 36-48. doi:10.1038/mp.2010.109

Wray, N. R., Ripke, S., Mattheisen, M., Trza skowski, M., Byme, E. M., Abdella oui, A., ... Major Depressive Disorder Working Group of the Psychiatric Genomics, C. (2018). Genomewide association analyses identify 44 risk va riants and refine the genetic architecture of major depression. Nat Genet, 50(5), 668-681. doi:10.1038/s41588-018-0090-3

Yang, J., Ba kshi, A., Zhu, Z, Hemani, G., Vinkhuyzen, A. A., Nolte, I. M., ... Visscher, P. M. (2015). Genome-wide genetic homogeneity between sexes and populations for human height and body mass index. Hum Mol Genet, 24(25), 7445-7449. doi:10.1093/hmg/ddv443

Yang, J ., Benya min, B., Mc Evoy, B. P., Gordon, S., Henders, A. K., Nyholt, D. R., . . Visscher, P. M. (2010). Common SNPs expla in a large proportion of the heritability for human height. Nat Genet, 42(7), 565-569. doi:10.1038/ng.608

Zaitlen, N., Lindstrom, S., Pasa niuc, B., Comelis, M., Genovese, G., Pollack, S., ... Price, A. L. (2012). Informed conditioning on c linic al cova ria tes increa ses power in case-control association studies. PLoS Genet, 8(11), e1003032. doi:10.1371/joumal.pgen.1003032

This article is protected by copyright. All rights reserved. 


\section{University Library}

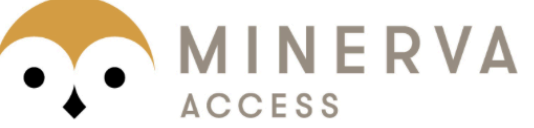

A gateway to Melbourne's research publications

Minerva Access is the Institutional Repository of The University of Melbourne

\section{Author/s:}

Trzaskowski, M;Mehta, D;Peyrot, WJ;Hawkes, D;Davies, D;Howard, DM;Kemper, KE;Sidorenko, J;Maier, R;Ripke, S;Mattheisen, M;Baune, BT;Grabe, HJ;Heath, AC;Jones, L;Jones, I;Madden, PAF;McIntosh, AM;Breen, G;Lewis, CM;Borglum, AD;Sullivan, PF;Martin, NG;Kendler, KS;Levinson, DF;Wray, NR

Title:

Quantifying between-cohort and between-sex genetic heterogeneity in major depressive disorder

Date:

2019-09-01

\section{Citation:}

Trzaskowski, M., Mehta, D., Peyrot, W. J., Hawkes, D., Davies, D., Howard, D. M., Kemper, K. E., Sidorenko, J., Maier, R., Ripke, S., Mattheisen, M., Baune, B. T., Grabe, H. J., Heath, A. C., Jones, L., Jones, I., Madden, P. A. F., McIntosh, A. M., Breen, G. ,... Wray, N. R. (2019). Quantifying between-cohort and between-sex genetic heterogeneity in major depressive disorder. AMERICAN JOURNAL OF MEDICAL GENETICS PART B-NEUROPSYCHIATRIC GENETICS, 180 (6), pp.439-447. https://doi.org/10.1002/ ajmg.b.32713.

Persistent Link:

http://hdl.handle.net/11343/285389 